\title{
A rare case of isolated splenic sarcoidosis: A case report and literature review
}

\author{
KAZUKI KOBAYASHI $^{1}$, TAKAHIRO EINAMA ${ }^{1}$, IBUKI FUJINUMA ${ }^{1}$, TAKAZUMI TSUNENARI ${ }^{1}$, \\ YOICHI MIYATA ${ }^{1}$, TOSHIMITSU IWASAKI ${ }^{1}$, JUNICHI ASAKUMA ${ }^{2}$, SHIGEYOSHI SOGA ${ }^{3}$, \\ HIROSHI SHINMOTO $^{3}$, HIRONORI TSUJIMOTO ${ }^{1}$, KAZUO HASE ${ }^{1}$, HIDEKI UENO ${ }^{1}$ and YOJI KISHI ${ }^{1}$
}

Departments of ${ }^{1}$ Surgery, ${ }^{2}$ Urology and ${ }^{3}$ Radiology, National Defense Medical College, Tokorozawa, Saitama 359-8513, Japan

Received March 13, 2020; Accepted October 14, 2020

DOI: $10.3892 / \operatorname{mco} .2020 .2184$

\begin{abstract}
Sarcoidosis is a multisystemic granulomatous disease. It is rarely isolated in the spleen. The present report describes a case of isolated splenic sarcoidosis that was diagnosed histologically following laparoscopic splenectomy. A 76-year-old woman, who underwent radical nephroureterectomy 7 years earlier for left renal pelvic cancer and mastectomy 6 years earlier for left breast cancer in another facility, was referred to our hospital for assessment of splenic tumors that were identified during a follow-up examination. The computed tomography scans revealed multiple nodules in the spleen, which had increased in size over 2 years. Positron emission tomography revealed accumulation of [18F]-fluorodeoxyglucose in the spleen. Laparoscopic splenectomy was performed and the diagnosis of sarcoidosis was confirmed histologically. A review of previous reports and the present case suggested that diagnosis of splenic sarcoidosis should be considered when the CT scans show multinodular splenic tumors, and sarcoidosis might be associated with malignant tumors.
\end{abstract}

\section{Introduction}

Sarcoidosis is a multisystemic granulomatous disease characterized by a chronic inflammatory process of an unidentified etiology. The most frequently affected organ is the lung, which accounts for $90 \%$ of all cases of sarcoidosis, followed by eyes, heart and lymphatic system (1). Splenic sarcoidosis is rare

Correspondence to: Dr Takahiro Einama, Department of Surgery, National Defense Medical College, 3-2 Namiki, Tokorozawa, Saitama 359-8513, Japan

E-mail: titiuehahaue@hotmail.com

Abbreviations: CT, computed tomography; FDG, fluorodeoxyglucose; LDH, lactate dehydrogenase; MRI, magnetic resonance imaging; PET, positron emission tomography; sIL-2R, soluble interleukin-2 receptor

Key words: sarcoidosis, multinodular splenic tumors, isolated splenic sarcoidosis and has been reported sporadically (2-12). Therefore, how to treat, diagnose, and manage isolated splenic sarcoidosis is not established.

Malignant lymphomas, lymphangiomas, or hemangiomas are the most frequently encountered splenic tumors. There are no specific radiological findings for sarcoidosis. So, differentiation between these tumors using radiological images alone is difficult; histological examination is usually required.

This report presents a case of isolated splenic sarcoidosis that was diagnosed histologically following laparoscopic splenectomy, because it was difficult to diagnose sarcoidosis using radiological images and laboratory tests. We also attempted to determine whether there are distinctive characteristics that could be associated with the diagnosis of splenic sarcoidosis.

\section{Case report}

A 76-year-old woman with a past medical history of left radical nephroureterectomy for left renal pelvic cancer 7 years previously and breast mastectomy for left breast cancer 6 years previously had been undergoing follow-up. Contrast-enhanced computed tomography (CT) revealed multiple poorly enhanced splenic tumors, with the largest one measuring $1.2 \mathrm{~cm}$ in diameter (Fig. 1). Using magnetic resonance imaging (MRI), the lesions showed equivalent intensity with the spleen on T1 weighted images and low intensity on T2-weighted images (Fig. 2). The lesions had increased in size over the previous 2 years. Positron emission tomography (PET)-CT revealed [18F]-fluorodeoxyglucose (FDG) accumulation at the upper and lower poles of the spleen (Fig. 3).

Because laboratory data showed slightly elevated lactate dehydrogenase (LDH; 275U/1) and soluble interleukin-2 receptor (sIL-2R; $588 \mathrm{U} / \mathrm{ml}$ ), we suspected that the lesions were malignant lymphoma. To confirm the diagnosis, we performed laparoscopic splenectomy. The operative time was 264 minutes, and the amount of bleeding was $291 \mathrm{ml}$. The patient recovered uneventfully and was discharged on the 8th postoperative day. Histological examination of the splenic specimen revealed small and dense epithelioid non-caseating granulomas (Fig. 4A). There was a marginal zone around the lymphoid follicles (Fig. 4B), but the targetoid pattern was negative for Ki-67 staining (Fig. 4C). Therefore, the diagnosis 

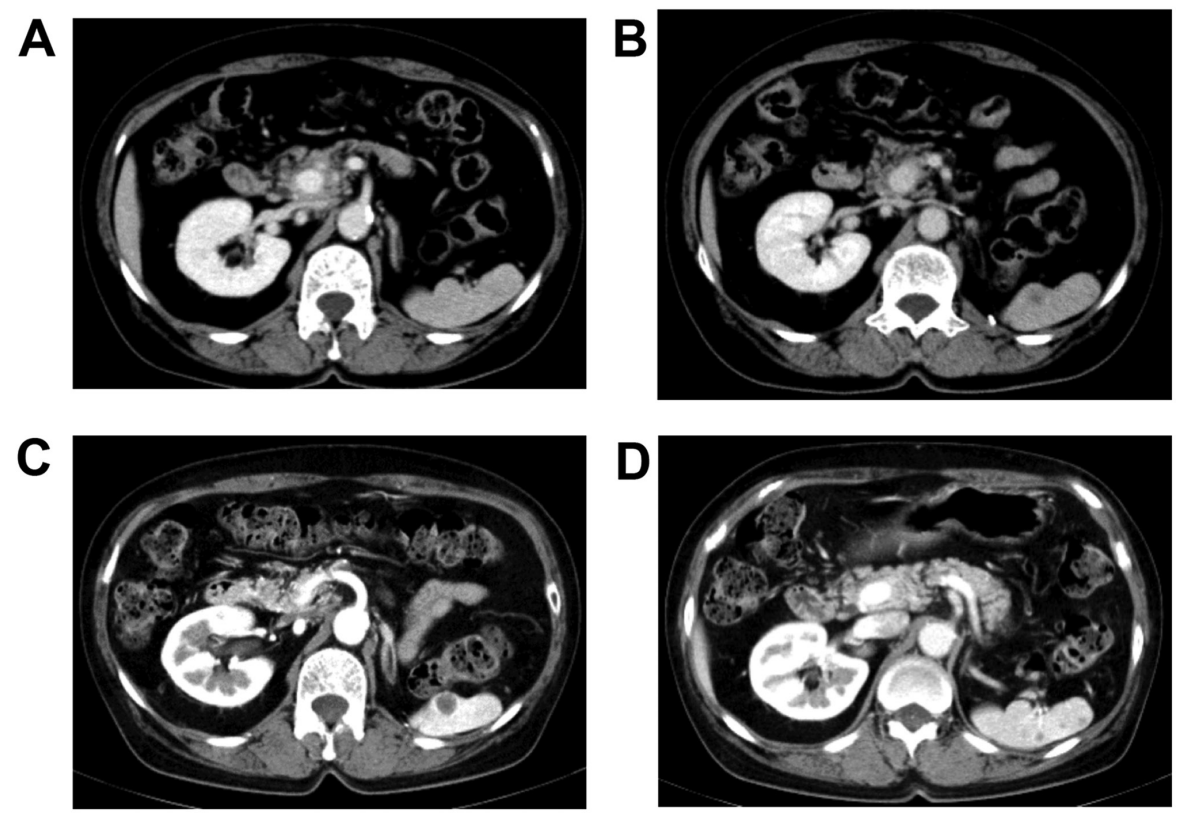

Figure 1. Contrast-enhanced CT (A) 2 years before, (B) 1 year before and (C) 7 months before splenectomy, revealing a gradual increase in size of the splenic tumor. (D) The tumor was multinodular (7 months before splenectomy).
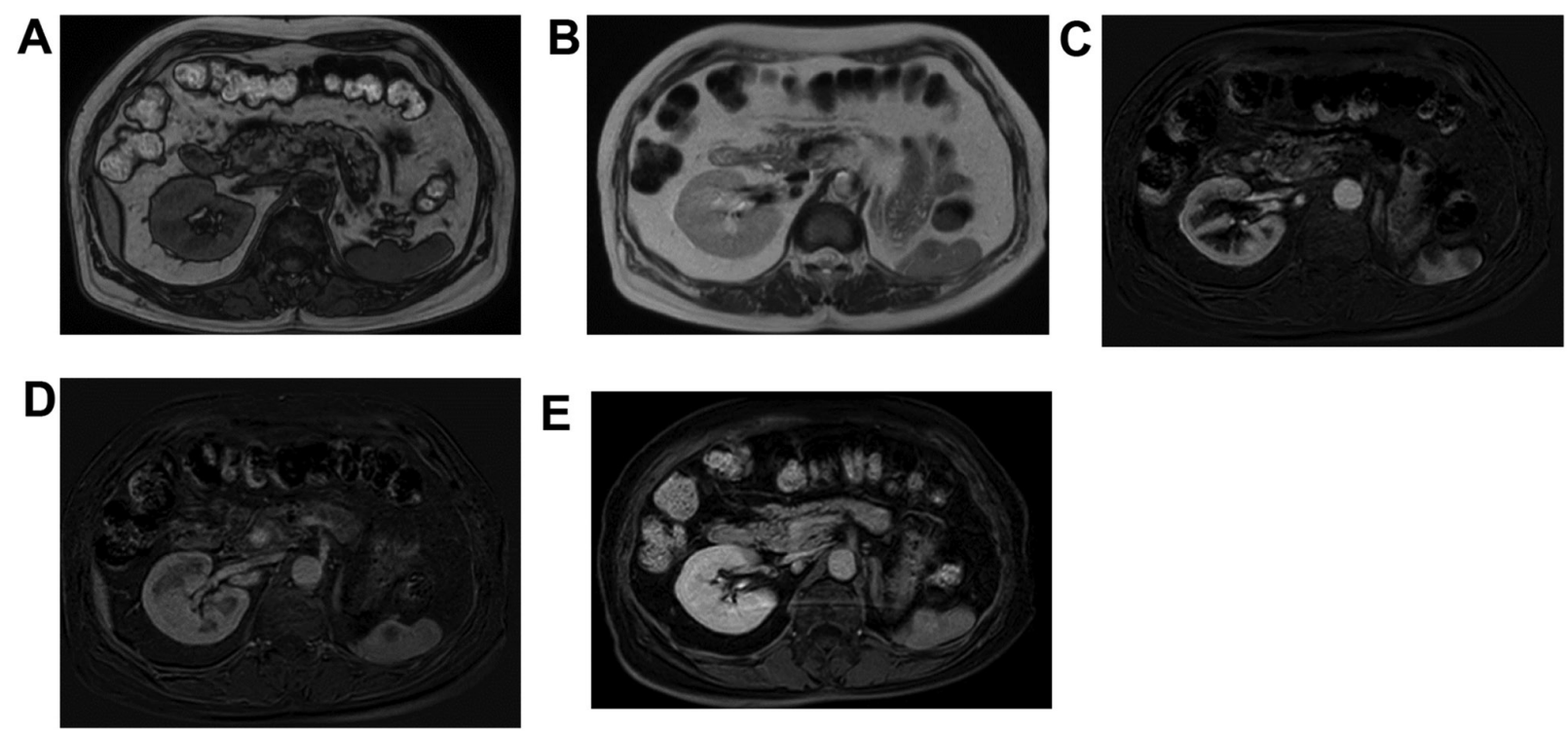

Figure 2. MRI of (A) T1 weighted image, (B) T2 weighted image, and dynamic study images of (C) arterial,(D) portal and (E) delayed phase revealing gradual increase of intensity in the splenic tumor.
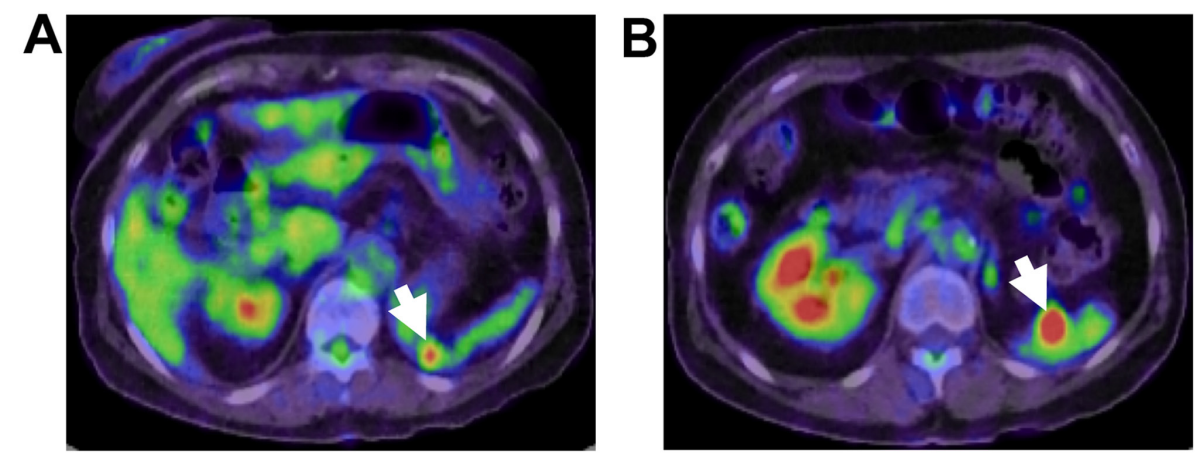

Figure 3. Positron emission tomography-CT revealed 18F-fluorodeoxyglucose accumulation at (A) upper and (B) lower poles of the spleen. White arrows indicate the accumulation of 18F-FDG at the upper and lower poles of the spleen. 
A

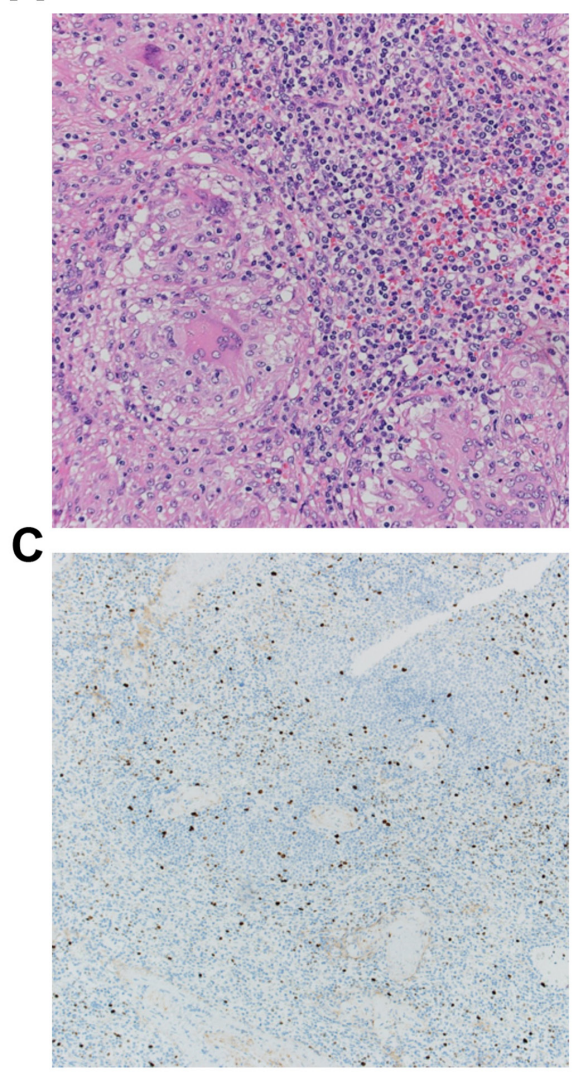

B

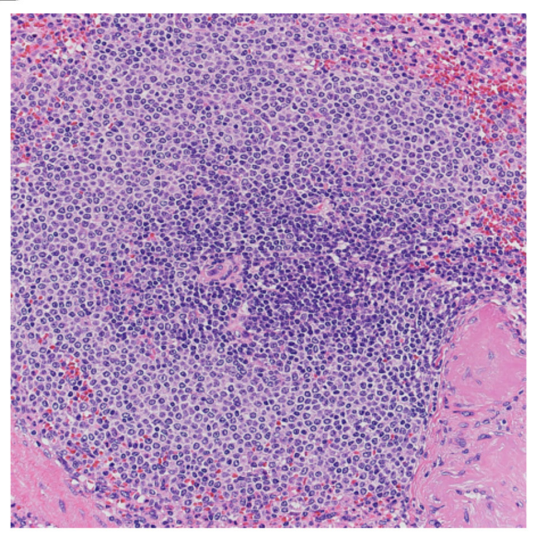

Figure 4. Histopathological findings of the tumor. (A) Granulomas (clusters of histiocytes and multinucleated cells) with fibrosis and lymphocytic infiltration. Magnification x40. (B) Splenic lymphoid follicles had germinal centers, accompanied by marginal zones. This finding suggested marginal zone lymphoma. Magnification x40. (C) Targetoid pattern, which is characteristic of marginal zone lymphoma, was negative. Magnification, x20.

of marginal zone lymphoma was not supported. Furthermore, microorganisms were not identified via Gram staining or Ziehl-Neelsen staining. Flow cytometry also did not confirm the diagnosis of malignant lymphoma. Postoperative laboratory test results demonstrated no remarkable change in $\mathrm{LDH}$ ( $235 \mathrm{U} / \mathrm{l})$ or sIL-2R $(565 \mathrm{U} / \mathrm{ml})$. Based on these tests, isolated sarcoidosis of the spleen was confirmed, although sarcoidosis was not detected in the patient's eyes, lungs, or heart. The patient remained alive after the 7 th month follow-up without signs of exacerbation of the sarcoidosis or recurrence of renal or breast cancer.

\section{Discussion}

We experienced a case of isolated splenic sarcoidosis. Regarding the affected organs of sarcoidosis, the spleen reportedly accounted for only $6.7 \%$ of occurrences and in most cases, other organs were involved (1). Isolated splenic sarcoidosis is rare and is reported sporadically (13). So diagnosis, treatment, and management of isolated splenic sarcoidosis was not established.

Sarcoidosis is an inflammatory disease characterized by the presence of non-caseating granulomas. Its diagnosis is based on clinical and radiological findings, in addition to histologically confirmed epithelioid granulomas. Laboratory tests are not usually helpful. Because there are no specific radiological findings for splenic sarcoidosis, histopathological examination is mandatory for its definitive diagnosis.
If there is a lesion of the spleen, methods for obtaining tissues for histopathology include biopsy and splenectomy. Because biopsy has the risk of bleeding and dissemination, especially where the tumor is malignant, splenectomy is commonly considered.

Granulomas are not a specific finding of sarcoidosis. The differential diagnoses of splenic granulomatous lesions include infection, foreign material exposure with talc or beryllium, benign vascular tumor, metastatic tumor, lymphoma, and Langerhans cell histiocytosis (14). According to an international consensus statement (15), even if the histopathological findings are indicative of sarcoidosis, a local sarcoid reaction, which develops in different neoplastic and non-neoplastic diseases, at the site of the main lesion and/or in regional lymph nodes, must be excluded. The present case fulfilled the criteria of Statement on sarcoidosis; therefore, the diagnosis of isolated splenic sarcoidosis was confirmed.

To the best of our knowledge, there are only 11 cases of isolated splenic sarcoidosis in the literature (2-12). Table I shows these 11 cases and the present case. It was suggested that splenic sarcoidosis was more common in women, and that sweating, and weight loss were frequently identified as subjective symptoms (5 among 11 cases; $45 \%$ ).

The lesions are often numerous (9 among 11 cases; 81.8\%) and, CT showed low-density multiple nodules (5 among 10 cases; 50\%), and splenomegaly (4 among 10 cases; 40\%). PET-CT showed abnormal accumulation of [18F]-FDG (4 among 6 cases; $66.7 \%$ ). In 10 cases, splenectomy was performed 


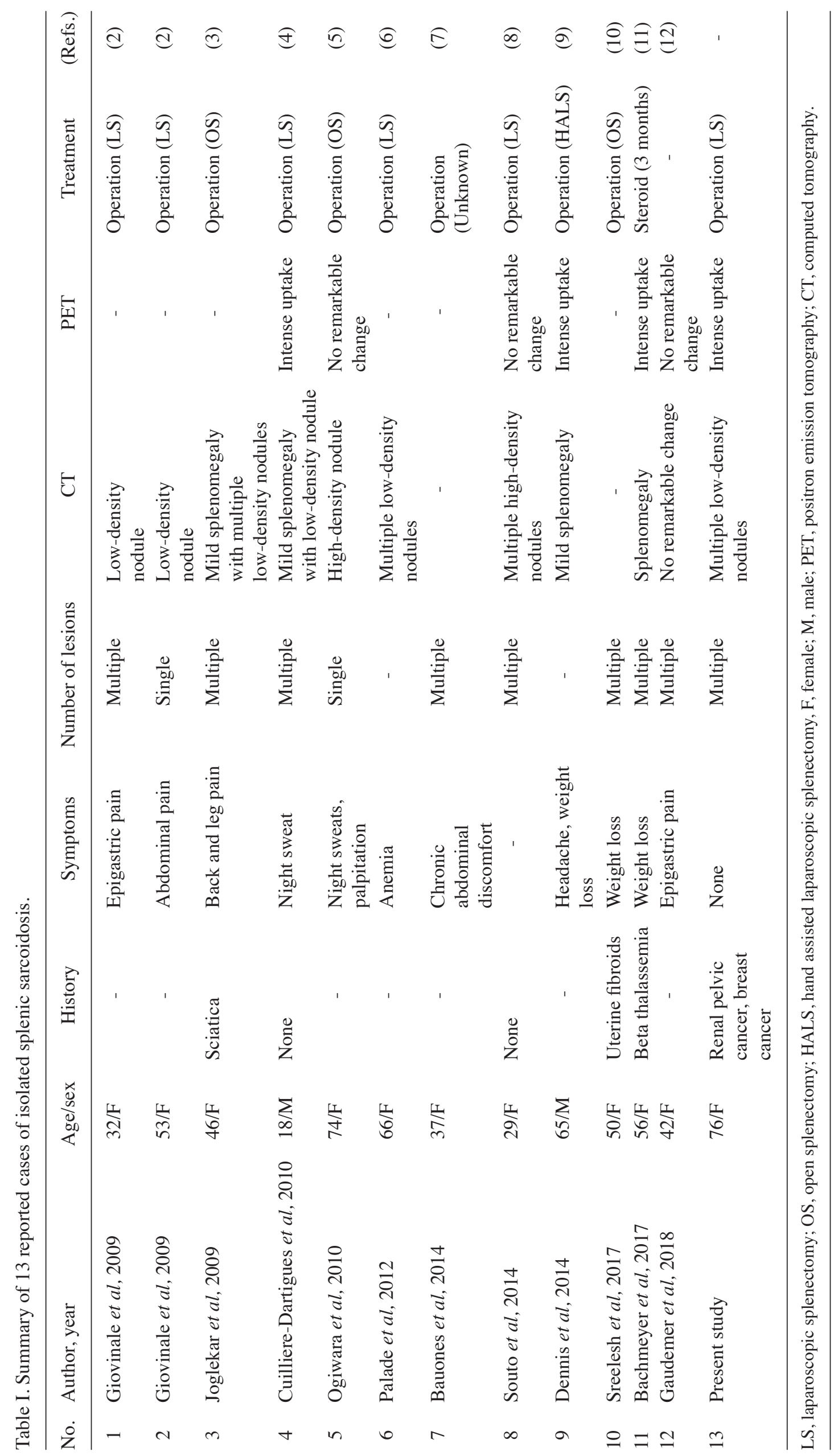


for the diagnosis. In one case, treatment with steroids were administered after confirmation of diagnosis.

In our case, for which the patient's past history was provided, included malignant tumor. The pathogenesis of sarcoid granulomas includes a complex interplay of immune cells, including macrophages dendritic cells, T helper lymphocytes, T regulatory cells, and their mediators. Although several studies have suggested that $\mathrm{T}$-cell receptor $\mathrm{V}$ beta, one of the subtypes of the T-cell antigen receptor, is associated with the conventional antigenic stimulation, the mechanism through which this stimulation causes sarcoidosis remains unclear (16). Malignant tumors might affect the body's immune system and be responsible for the development of sarcoidosis in the spleen, which is a hematopoietic lymphoid organ. There are no reports clarifying them, so further case accumulation might be necessary.

In conclusion, we experienced a rare case of isolated sarcoidosis of the spleen. Sarcoidosis should be included in the differential diagnosis when multiple splenic tumors are detected and sarcoidosis might be associated with malignant tumors.

\section{Acknowledgements}

The authors would like to thank Dr Hiroaki Aoki, Associate General Manager of the Surgical Department at Jikei University, for his kindness and for the fruitful discussion we had at the 852nd Annual Meeting of the Tokyo Surgical Society.

\section{Funding}

No funding was received.

\section{Availability of data and materials}

The datasets used and/or analyzed during the current study available from the corresponding author on reasonable request.

\section{Authors' contributions}

KK and TE were involved in drafting the manuscript, revising it critically for important intellectual content, and made substantial contributions to acquisition of data. IF, TT and JA analyzed and interpreted the patient data, and contributed to manuscript preparation. SS and HS made substantial contributions to analysis and interpretation of data. YM and TI made substantial contributions to conception and design. YK, HT, $\mathrm{KH}$ and $\mathrm{HU}$ made substantial contributions to conception and design, and gave final approval of the version to be published. All authors read and approved the final manuscript.

\section{Ethics approval and consent to participate}

This case report was approved by the Institutional Review Board of the National Defense Medical College (approval no. 4115).

\section{Patient consent for publication}

Written informed consent for publication of their clinical details and/or clinical images was obtained from the patient.

\section{Competing interests}

The authors declare that they have no competing interests.

\section{References}

1. Baughman RP, Teirstein AS, Judson MA, Rossman MD, Yeager H Jr, Bresnitz EA, DePalo L, Hunninghake G, Iannuzzi MC, Johns CJ, et al: Clinical characteristics of patients in case control study of sarcoidosis. Am J Respir Crit Care Med 164: 1885-1889, 2001.

2. Giovinale M, Fonnesu C, Soriano A, Cerquaglia C, Curigliano V, Verrecchia E, De Socio G, Gasbarrini G and Manna R: Atypical sarcoidosis: Case reports and review of the literature. Eur Rev Med Pharmacol Sci 13: 37-44, 2009.

3. Joglekar SP, Hudson RL, Lgasundaram R and Pereira JH: 'Surgical cure' for non parathyroid hypercalcemia. World J Surg Oncol 7: 23, 2009

4. Cuilliere-Dartigues P, Meyohas MC, Balladur P, Gorin NC and Coppo P: Splenic sarcoidosis: An unusual aetiology of agranulocytosis. Am J Hematol 85: 891, 2010.

5. Ogiwara Y, Mori S, Iwama M, Sawabe M, Kanazawa N, Takemoto M, Kanazawa N, Fukuda I, Kondo Y, Kimbara Y, et al: Hypoglycemia due to ectopic secretion of insulin-like growth factor-I in a patient with an isolated sarcoidosis of the spleen. Endocr J 57: 325-330, 2010.

6. Palade R, Voiculescu D, Suliman E and Simion G: Splenic sarcoidosis-a case report. Chirurgia (Bucur) 107: 670-674, 2012.

7. Bauones S, Le Corroller T, Durieux O, Guenoun D, Del Grande J, Pirro N and Champsaur P: Splenic sarcoidosis mimicking neoplastic disease. J Clin Ultrasound 42: 38-41, 2014.

8. Souto MM, Tempes BC, Lambert BF Trindade EN and Trindade MR: Laparoscopic splenectomy for isolated splenic sarcoidosis. JSLS 18: 155-159, 2014.

9. Dennis BA, Jajosky RP and Harper RJ: Splenic sarcoidosis without focal nodularity: A case of 1,25-dihydroxyvitamin D-mediated hypercalcemia localized with FDG PET/CT. Endocr Pract 20: e28-e33, 2014.

10. Sreelesh KP, Kumar ML and Anoop TM: Primary splenic sarcoidosis. Proc (Bayl Univ Med Cent) 27: 344-345, 2014.

11. Bachmeyer C, Fayand A, Georgin-Lavialle S, Fedida B, Naccache JM, Lionnet F and Amiot X: Massive splenomegaly indicating sarcoidosis. Am J Med 130: e141-e142, 2017.

12. Gaudemer A, Sauvet G, Hij A, Stanciu R, Farge-Bancel D and Algayres JP: Splenic sarcoidosis diagnosed by US-guided biopsy: About a case. Rev Med Interne 39: 200-202, 2018 (In French).

13. Warshauer DM and Lee JK: Imaging manifestations of abdominal sarcoidosis. AJR Am J Roentgenol 182: 15-28, 2004.

14. O'Maley DP, George TI, Orazi A and Abbondanzo SL: Atlas of Nontumor Pathology Benign and Reactive Conditions of Lymph Node and Spleen. America Amer Registry of Pathology, 2009.

15. Statement on sarcoidosis. Joint statement of the American thoracic society (ATS), the European respiratory society (ERS) and the world association of sarcoidosis and other granulomatous disorders (WASOG) adopted by the ATS Board of Directors and by the ERS Executive Committee, February 1999. Am J Respir Crit Care Med 160: 736-755, 1999.

16. Andrew F and Talmadge E; Pathology and pathogenesis of sarcoidosis. https://www.uptodate.com/contents/ pathology-and-pathogenesis-of-sarcoidosis?search=Pathology $\%$ 20 and $\% 20$ pathogenesis $\% 20$ of $\% 20$ sarcoidosis \&source=search result\&selectedTitle $=1 \sim 150 \&$ usage_type $=$ default\&display_rank $=1$. Accessed 09 Apr 2018. 